\title{
Constraints on the Neutrino Mass from Cosmology and their impact on world neutrino data.
}

\author{
A. Melchiorri ${ }^{\mathrm{a}}$, G.L. Fogli, E. Lisi, A. Marrone, A. Palazzo ${ }^{\mathrm{b}}$, P. Serra ${ }^{\mathrm{c}}$ and J. Silk ${ }^{\mathrm{d}}$ \\ aDipartimento di Fisica and Sezione INFN, Università degli Studi di Roma "La Sapienza", P.le Aldo \\ Moro 5, 00185, Rome, Italy

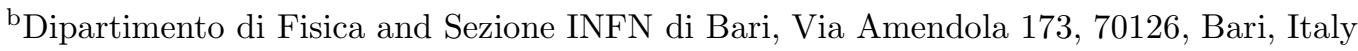 \\ cDipartimento di Fisica, Università degli Studi di Roma "La Sapienza", P.le Aldo Moro 5, 00185, Rome, \\ Italy \\ dAstrophysics, Denys Wilkinson Building, Keble Road, OX13RH,Oxford, United Kingdom
}

We derive upper limits on the sum of neutrino masses from an updated combination of data from Cosmic Microwave Background experiments and Galaxy Redshifts Surveys. The results are discussed in the context of three-flavor neutrino mixing and compared with neutrino oscillation data, with upper limits on the effective neutrino mass in Tritium beta decay from the Mainz and Troitsk experiments and with the claimed lower bound on the effective Majorana neutrino mass in neutrinoless double beta decay from the Heidelberg-Moscow experiment.

\section{Introduction}

Cosmological observations have started to provide valuable upper limits on absolute neutrino masses (see, e.g., the reviews 12]), competitive with those from laboratory experiments. In particular, the combined analysis of high-precision data from Cosmic Microwave Background (CMB) anisotropies and Large Scale Structures (LSS) has already reached a sensitivity of $O(\mathrm{eV})$ (see, e.g., 345 ) for the sum of the neutrino masses $\Sigma$,

$\Sigma=m_{1}+m_{2}+m_{3}$.

We recall that the total neutrino energy density in our Universe, $\Omega_{\nu} h^{2}$ (where $h$ is the Hubble constant normalized to $H_{0}=100 \mathrm{~km} \mathrm{~s}^{-1} \mathrm{Mpc}^{-1}$ ) is related to $\Sigma$ by the well-known relation $\Omega_{\nu} h^{2}=$ $\Sigma /(93.2 \mathrm{eV})$ [6], and plays an essential role in theories of structure formation. It can thus leave key signatures in LSS data (see, eg., [7]) and, to a lesser extent, in CMB data (see, e.g., 8]). Very recently, it has also been shown that accurate Lyman- $\alpha(\operatorname{Ly} \alpha)$ forest data 9], taken at face value, can improve the current CMB+LSS constraints on $\Sigma$ by a factor of $\sim 3$, with important consequences on absolute neutrino mass scenarios 10 .

On the other hand, atmospheric, solar, reactor and accelerator neutrino experiments have convincingly established that neutrinos are massive and mixed. World neutrino data are consistent with a three-flavor mixing framework (see [1] and references therein), parameterized in terms of three neutrino masses $\left(m_{1}, m_{2}, m_{3}\right)$ and of three mixing angles $\left(\theta_{12}, \theta_{23}, \theta_{13}\right)$, plus a possible $\mathrm{CP}$ violating phase $\delta$.

Neutrino oscillation experiments are sensitive to two independent squared mass difference, $\delta m^{2}$ and $\Delta m^{2}$ (with $\delta m^{2} \ll \Delta m^{2}$ ), hereafter defined as 12 ]

$\left(m_{1}^{2}, m_{2}^{2}, m_{3}^{2}\right)=\mu^{2}+\left(-\frac{\delta m^{2}}{2},+\frac{\delta m^{2}}{2}, \pm \Delta m^{2}\right)$,

where $\mu$ fixes the absolute neutrino mass scale, while the cases $+\Delta m^{2}$ and $-\Delta m^{2}$ identify the so-called normal and inverted neutrino mass hierarchies, respectively. Neutrino oscillation data indicate that $\delta m^{2} \simeq 8 \times 10^{-5} \mathrm{eV}^{2}$ and $\Delta m^{2} \simeq$ $2.4 \times 10^{-3} \mathrm{eV}^{2}$. They also indicate that $\sin ^{2} \theta_{12} \simeq$ $0.3, \sin ^{2} \theta_{23} \simeq 0.5$, and $\sin ^{2} \theta_{13} \leq$ few $\%$. However, they are currently unable to determine the mass hierarchy $\left( \pm \Delta m^{2}\right)$ and the phase $\delta$, and are 
insensitive to the absolute mass parameter $\mu$ in Eq. (2).

The absolute neutrino mass scale can also be probed by non-oscillatory neutrino experiments. The most sensitive laboratory experiments to date have been focussed on tritium beta decay and on neutrinoless double beta decay. Beta decay experiments probe the so-called effective electron neutrino mass $m_{\beta}$ [13],

$m_{\beta}=\left[c_{13}^{2} c_{12}^{2} m_{1}^{2}+c_{13}^{2} s_{12}^{2} m_{2}^{2}+s_{13}^{2} m_{3}^{2}\right]^{\frac{1}{2}}$,

where $c_{i j}^{2}=\cos ^{2} \theta_{i j}$ and $s_{i j}^{2}=\sin ^{2} \theta_{i j}$. Current experiments (Mainz [14] and Troitsk [15]) provide upper limits in the range $m_{\beta} \leq$ few eV [16].

Neutrinoless double beta decay $(0 \nu 2 \beta)$ experiments are instead sensitive to the so-called effective Majorana mass $m_{\beta \beta}$ (if neutrinos are Majorana fermions),

$m_{\beta \beta}=\left|c_{13}^{2} c_{12}^{2} m_{1}+c_{13}^{2} s_{12}^{2} m_{2} e^{i \phi_{2}}+s_{13}^{2} m_{3} e^{i \phi_{3}}\right|$,

where $\phi_{2}$ and $\phi_{3}$ parameterize relative (and unknown) Majorana neutrino phases 17. All $0 \nu 2 \beta$ experiments place only upper bounds on $m_{\beta \beta}$ (the most sensitive being in the $\mathrm{eV}$ range, with the exception of the Heidelberg-Moscow experiment 18, which claims a positive (but highly debated) $0 \nu 2 \beta$ signal $m_{\beta \beta}>0.17 \mathrm{eV}$ at $95 \%$ c.l. and corresponding to $m_{\beta \beta}$ in the sub-eV range at best fit 1920 .

In these proceedings, we will briefly illustrate the impact of the cosmological constraints on the sum of neutrino masses on the three-flavor mixing theoretical and observational scenario.

\section{Upper bounds on $\Sigma$ from cosmological data}

The neutrino contribution to the overall energy density of the universe can play a relevant role in large scale structure formation and leave key signatures in several cosmological data sets. More specifically, neutrinos suppress the growth of fluctuations on scales below the horizon when they become non relativistic. A massive neutrinos of a fraction of $\mathrm{eV}$ would therefore produce a significant suppression in the clustering on small cosmological scales (namely, for comoving wavenumber $\left.k \sim 0.05 h \mathrm{Mpc}^{-1}\right)$.
To constrain $\Sigma$ from cosmological data, we perform a likelihood analysis comparing the recent observations with a set of models with cosmological parameters sampled as follows: cold dark matter $(\mathrm{cdm})$ density $\Omega_{\mathrm{cdm}} h^{2} \in[0.05,0.20]$ in steps of 0.01; baryon density $\Omega_{b} h^{2} \in[0.015,0.030]$ (motivated by Big Bang Nucleosynthesis) in steps of 0.001 ; a cosmological constant $\Omega_{\Lambda} \in[0.50,0.96]$ in steps of 0.02 ; and neutrino density $\Omega_{\nu} h^{2} \in$ $[0.001,0.020]$ in steps of 0.002 . We restrict our analysis to flat $\Lambda$-CDM models, $\Omega_{\mathrm{tot}}=1$, and we add a conservative external prior on the age of the universe, $t_{0}>10$ Gyrs. The value of the Hubble constant in our database is not an independent parameter, since it is determined through the flatness condition. We adopt the conservative top-hat bound $0.50<h<0.90$ and we also consider the $1 \sigma$ constraint on the Hubble parameter, $h=0.71 \pm 0.07$, obtained from Hubble Space Telescope (HST) measurements [21]. We allow for a reionization of the intergalactic medium by varying the CMB photon optical depth $\tau_{c}$ in the range $\tau_{c} \in[0.05,0.30]$ in steps of 0.02 .

We restrict the analysis to adiabatic inflationary models with a negligible contribution of gravity waves. We let vary the spectral index $n$ of scalar primordial fluctuations in the range $n \in$ $[0.85,1.3]$ and its running $d n / d \ln k \in[-0.40,0.2]$ assuming pivot scales at $k_{0}=0.05 \mathrm{Mpc}^{-1}$ and $k_{0}=0.002 \mathrm{Mpc}^{-1}$. We rescale the fluctuation amplitude by a prefactor $C_{110}$, in units of the value $C_{110}^{\text {WMAP }}$ measured by the Wilkinsin Microwave Anisotropy Probe (WMAP) satellite. Finally, concerning the neutrino parameters, we fix the number of neutrino species to $N_{\nu}=3$, all with the same mass (the effect of mass differences compatible with neutrino oscillation being negligible in the current cosmological data 22]). An higher number of neutrino species can weakly affect both CMB and LSS data (see, e.g., 23]) but is highly constrained by standard big bang nucleosynthesis and is not considered in this work, where we focus on $3 \nu$ mixing.

The cosmological data we considered comes from observation of CMB anisotropies and polarization, galaxy redshift surveys and luminosity distances of type Ia supernovae. For the CMB data we use the recent temperature and cross po- 
larization results from the WMAP satellite [3] using the method explained in 24 and the publicly available code. Given a theoretical temperature anisotropy and polarization angular power spectrum in our database, we can therefore associate a $\chi_{\mathrm{WMAP}}^{2}$ to the corresponding theoretical model.

We further include the latest results from other CMB datasets. The CMB data analysis methods have been already described in [11] and will not be reported here.

In addition to the CMB data we also consider the real-space power spectrum of galaxies from either the 2 degrees Fields (2dF) Galaxy Redshifts Survey or the Sloan Digital Sky Survey (SDSS), using the data and window functions of the analysis of [25] and [4. We restrict the analysis to a range of scales over which the fluctuations are assumed to be in the linear regime $\left(k<0.2 h^{-1} \mathrm{Mpc}\right)$. When combining with the CMB data, we marginalize over a bias $b$ for each data set considered as an additional free parameter.

We also include information from the Ly $\alpha$ Forest in the SDSS, using the results of the analysis of [10] and 9], which probe the amplitude of linear fluctuations at very small scales. For this data set, small-scale power spectra are computed at high redshifts and compared with the values presented in 9]. As in [10, we do not consider running.

We finally incorporate constraints obtained from the SN-Ia luminosity measurements of [26] using the so-called GOLD data set. Luminosity distances at SN-Ia redshifts are computed for each model in our database and compared with the observed apparent bolometric SN-Ia luminosities. In Fig. 1 we plot the likelihood distribution for $\Sigma$ from our joint analysis of CMB $+\mathrm{SN}-$ $\mathrm{Ia}+\mathrm{HST}+$ LSS data, transformed into an equivalent $\Delta \chi_{\Sigma}^{2}$ function, which allows to derive bounds on $\Sigma$ at any fixed confidence level. We take LSS data either from the SDSS or the $2 \mathrm{dF}$ survey (dashed and solid curves, respectively). ${ }^{1}$

As we can see, these curves do not show evidence for a neutrino mass (the best fit being at

\footnotetext{
${ }^{1}$ For the sake of brevity, the subdominant block of data
} $(\mathrm{SN}-\mathrm{Ia}+\mathrm{HST})$ is not explicitly indicated in figure labels.
$\Sigma \simeq 0)$ and provide the $2 \sigma$ bound $\Sigma \leq 1.4 \mathrm{eV}$. Such bound is in good agreement with previous results in similar analyses 32742829 .

Also plotted in Fig. 1 is the $\Delta \chi_{\Sigma}^{2}$ function from a joint analysis of $\mathrm{CMB}+\mathrm{SN}-$ $\mathrm{Ia}+\mathrm{HST}+2 \mathrm{dF}+$ Ly $\alpha$. No running is assumed in this analysis, and we find a $2 \sigma$ bound $\Sigma<0.47$ $\mathrm{eV}$, in very good agreement (despite the more approximate method we used) with the analysis already presented in 10 .

As shown in Fig. 1 and already discussed in 10, the inclusion of the Ly $\alpha$ data from the SDSS set greatly improves the constraints on $\Sigma$.

\section{Adding bounds from laboratory and As- trophysics}

Here we consider confidence regions obtained from analysis of neutrino oscillation data, of $m_{\beta}$ and $m_{\beta \beta}$ data and cosmological CMB+LSS data (see 11] for more details).

Figure 2 shows such regions projected in the three coordinate planes. Separate laboratory and cosmological upper bounds at the $2 \sigma$ level are shown as dashed lines, while the regions allowed by the combination of laboratory, cosmological, and oscillation data are shown as thick solid curves for normal hierarchy and as thin solid curves for inverted hierarchy. It can be seen that the upper bounds on the $\left(m_{\beta}, m_{\beta \beta}, \Sigma\right)$ observables are dominated by the cosmological upper bound on $\Sigma$. This bound, via the $\left(m_{\beta}, \Sigma\right)$ and $\left(m_{\beta \beta}, \Sigma\right)$ correlations induced by oscillation data, provides upper limits also on $m_{\beta \beta}$ and $m_{\beta}$, which happen to be stronger than the current laboratory limits by a factor $\sim 4$.

Since significant improvements on laboratory limits for $m_{\beta \beta}$ and $m_{\beta}$ will require new experiments and several years of data taking [16], cosmological determinations of $\Sigma$, although indirect, will continue to provide, in the next future, the most sensitive upper limits (and hopefully a signal) for absolute neutrino mass observables.

In Fig. 2, the tension (at $2 \sigma$ ) between the limits from cosmology and the lower limit on $m_{\beta \beta}>$ $0.17 \mathrm{eV}$ claimed by the Heidelberg-Moscow experiment is a clear symptom of possible problems, either in some data sets or in their theoretical in- 


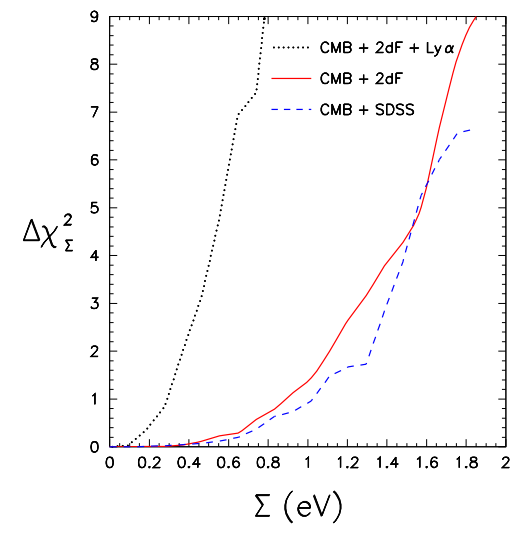

Figure 1. Upper bounds on the sum of neutrino masses $\Sigma$ from our $3 \nu$ analysis of cosmological data, given in terms of the $\Delta \chi_{\Sigma}^{2}$ function. The solid and dashed curves refer to the combination of $\mathrm{CMB}$ and LSS data $(\mathrm{CMB}+2 \mathrm{dF}$ and CMB+SDSS, respectively). The two CMB+LSS fits provide comparable results and, for definiteness, the $\mathrm{CMB}+2 \mathrm{df}$ one is adopted. In addition, we consider also the case where the recent Ly $\alpha$ data from the SDSS are included, providing significantly stronger constraints on $\Sigma$ (dotted curve). See 11 for details.

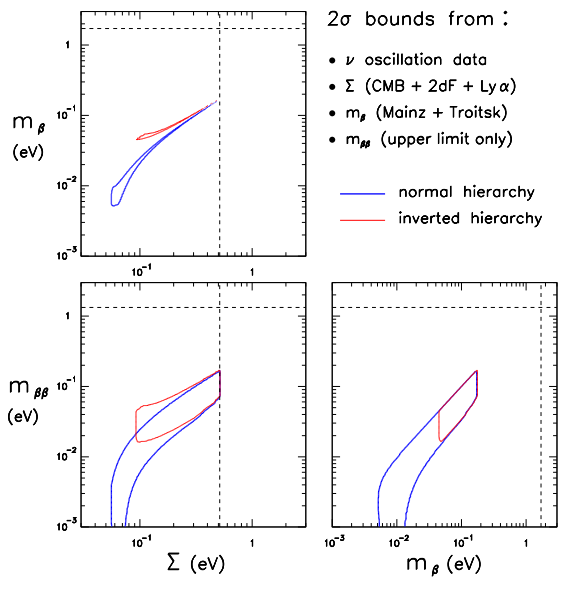

Figure 2. Global $3 \nu$ analysis in the $\left(m_{\beta}, m_{\beta \beta}, \Sigma\right)$ parameter space, using oscillation data plus laboratory data and cosmological data. This figure implements also upper limits (shown as dashed lines at $2 \sigma$ level) on $m_{\beta}$ from Mainz+Troitsk data, on $m_{\beta \beta}$ from $0 \nu 2 \beta$ data, and on $\Sigma$ from $\mathrm{CMB}+2 \mathrm{dF}+\mathrm{Ly} \alpha$ data. In combination with oscillation parameter bounds, the cosmological upper limit on $\Sigma$ dominates over the laboratory upper limits on $m_{\beta}$ and $m_{\beta \beta}$. See [1] for details. 
terpretation, which definitely prevent any global combination of data. It would be premature to conclude that, e.g., the $0 \nu 2 \beta$ claim is "ruled out" by cosmological data but it is anyway exciting that global neutrino data analyses have already reached a point where fundamental questions may start to arise.

Acknowledgements A.M. would like to thank the Organizers of the NOW-2004 workshop. The work of A.M. is supported by the Italian Ministero dell'Istruzione, Università e Ricerca (MIUR) through the "GEMINI" research project and Istituto Nazionale di Fisica Nucleare (INFN) through the "Astroparticle Physics" research project. The work of G.L.F., E.L., A.M.2, and A.P. is supported by the Italian Ministero dell'Istruzione, Università e Ricerca (MIUR) and Istituto Nazionale di Fisica Nucleare (INFN) through the "Astroparticle Physics" research project.

\section{REFERENCES}

1. V. Barger, D. Marfatia and K. Whisnant, Int. J. Mod. Phys. E 12, 569 (2003).

2. A.D. Dolgov, Phys. Rept. 370, 333 (2002).

3. WMAP Collaboration, C.L. Bennett et al., Astrophys. J. Suppl. 148, 1 (2003).

4. SDSS Collaboration, M. Tegmark et al., Phys. Rev. D 69, 103501 (2004).

5. See also O. Lahav, "Massive Neutrinos and Cosmology," in Neutrino 2004.

6. Review of Particle Physics, S. Eidelman et al., Phys. Lett. B 592, 1 (2004).

7. W. Hu, D.J. Eisenstein, and M. Tegmark, Phys. Rev. Lett. 80, 5255 (1998).

8. C.P. Ma and E. Bertschinger, Astrophys. J. 455, 7 (1995).

9. SDSS Collaboration, P. McDonald et al., astro-ph/0405013

10. U. Seljak et al., astro-ph/0407372

11. G. L. Fogli, E. Lisi, A. Marrone, A. Melchiorri, A. Palazzo, P. Serra and J. Silk, Phys. Rev. D 70 (2004) 113003 arXiv:hep-ph/0408045.

12. G. L. Fogli, E. Lisi, D. Montanino, and A. Palazzo, Phys. Rev. D 65, 073008 (2002).

13. B.H.J. McKellar, Phys. Lett. B 97, 93 (1980);
F. Vissani, Nucl. Phys. B (Proc. Suppl.) 100, 273 (2001); J. Studnik and M. Zralek, hep-ph/0110232 See also the discussion in Y. Farzan and A.Yu. Smirnov, Phys. Lett. B 557, 224 (2003).

14. C. Weinheimer, in the Proceedings of Neutrino 2002, p. 279.

15. V.M. Lobashev, in the Proceedings of NPDC 17, ed. by N. Auerbach, Zs. Fulop, Gy. Gyurky, and E. Somorjai, Nucl. Phys. A 719, 153 (2003).

16. K. Eitel, "Direct Neutrino Mass Experiments," in Neutrino 2004 [?].

17. S.M. Bilenky, J. Hosek, and S.T. Petcov, Phys. Lett. B 94, 495 (1980); J. Schechter and J.W.F. Valle, Phys. Rev. D 22, 2227 (1980).

18. H.V. Klapdor-Kleingrothaus, A. Dietz, H.L. Harney, and I.V. Krivosheina, Mod. Phys. Lett. A 16, 2409 (2001).

19. H.V. Klapdor-Kleingrothaus, A. Dietz, I.V. Krivosheina, and O. Chkvorets, Nucl. Instrum. Meth. A 522, 371 (2004).

20. H.V. Klapdor-Kleingrothaus, I.V. Krivosheina, A. Dietz, and O. Chkvorets, Phys. Lett. B 586, 198 (2004).

21. W. Freedman et al., Astrophysical Journal, 553, 47 (2001).

22. J. Lesgourgues, S. Pastor, and L. Perotto, Phys. Rev. D 70, 045016 (2004).

23. R. Bowen et al., Mon. Not. Roy. Astron. Soc. 334, 760 (2002).

24. L. Verde et al., Astrophys. J. Suppl. 148, 195 (2003).

25. W.J. Percival et al., Mon. Not. Roy. Astron. Soc. 327, 1297 (2001).

26. Supernova Search Team Collaboration, A.G. Riess et al., Astrophys. J. 607, 665 (2004).

27. S. Hannestad, JCAP 0305, 004 (2003).

28. V. Barger, D. Marfatia, and A. Tregre, Phys. Lett. B 595, 55 (2004).

29. P. Crotty, J. Lesgourgues, and S. Pastor, Phys. Rev. D 69, 123007 (2004). 